\title{
Enfoques disciplinarios e interdisciplinarios para el análisis y definición de la violencia*
}

\author{
[Versión en Castellano] \\ Disciplinary and Interdisciplinary Approaches to the Analysis and \\ Definition of Violence
}

Abordagens disciplinares e interdisciplinares para a análise e definição de violência

Recibido el 10 de octubre, 2019. Aceptado el 16 de marzo, 2020.

Tania Galaviz-Armenta**
https://orcid.org/0000-0001-5464-7641
México

\section{Resumen}

- Para citar este artículo:

Galaviz-Armenta, Tania (2021)

Enfoques disciplinarios e

interdisciplinarios para el análisis

y definición de la violencia.

Ánfora, 28(50), 161-182.

https://doi.org/10.30854/anfv28.n50.2021.656

Universidad Autónoma de

Manizales. ISSN 0121-6538/

e-ISSN 2248-6941.

CC BY-NC-SA 4.0

Objetivo: a pesar de la abundante discusión académica acerca de la violencia, sus formas, actores, efectos, entre otros elementos, una pregunta emerge casi de manera permanente: ¿de qué hablamos cuando hablamos de violencia? El presente artículo realiza una Reflexión sobre la definición y análisis del concepto de la violencia desde distintos enfoques disciplinarios e interdisciplinarios con el objetivo de intentar una respuesta para la pregunta ¿qué se entiende por violencia? Metodología: se consideraron algunos de los aportes realizados desde la Antropología, las Ciencias

\footnotetext{
* Este artículo de Reflexión hace parte de la Investigación "Conflicto, paz y violencias: procesos de participación social". Investigación desarrollada en la Universidad Autónoma del Estado de Morelos, México, Centro de Investigación en Ciencias Sociales y Estudios Regionales. La financiación del proyecto fue asumida por la investigadora, quien declara que no hubo conflicto de interés en la ejecución del proyecto de investigación.

** Doctora en Ciencias Políticas y Sociales. Profesora de la Universidad Autónoma del Estado de Morelos, México, Centro de Investigación en Ciencias Sociales y Estudios Regionales. Correo electrónico: tgalaviz@uaem.mx
} 
Políticas, la Filosofía, la Sociología, la Investigación para la Paz, la Criminología y la Salud Pública. Resultados: se encontró que la mayoría de los trabajos que sirvieron de base a este estudio consideran a la violencia como un elemento que delimita las interacciones sociales más que un acto irracional o instintivo. Y, dependiendo la perspectiva de análisis, establecen el grado de influencia de lo cultural, simbólico, institucional y normativo en su manejo y justificación. Conclusiones: se concluye con un balance de las ventajas y desventajas de la conveniencia analítica del tránsito del término violencia a la expresión violencias. Ello, considerando un enfoque interdisciplinario que no sólo se centra en las manifestaciones físicas, sino que atiende la multidimensionalidad de la violencia y el entramado que crean las distintas escalas de interacción y afectación convirtiéndola en fenómeno social mutable y complejo.

Palabras-clave: Violencia; Violencias; Análisis conceptual; Definiciones de violencia; Enfoques disciplinarios e interdisciplinarios.

\section{Abstract}

Objective: despite the plentiful academic discussion about violence, forms of violence, actors, effects, among other things, a question emerges almost permanently: what do we talk about when we talk about violence? This article makes a reflection on the definition and analysis of the concept of violence from different disciplinary and interdisciplinary approaches in order to answer the question: what is meant by violence? Methodology: some approaches from Anthropology, Political Science, Philosophy, Sociology, Research for Peace, Criminology and Public Health were considered. Results: it was found that most of the research considers violence as an element that delimits social interactions rather than an irrational or instinctive act. And, these studies establish the degree of cultural, symbolic, institutional influences and the normative in its management and reasoning, depending on the perspective of analysis. Conclusion. a review of advantages and disadvantages of the analytical expediency of the transition from the term violence into the expression of violences was concluded. It considers an interdisciplinary approach that not only focuses on physical manifestations, but addresses the multidimensionality of violence and the matter created by different scales of interaction and affectation by making the violence a changeable and complex social phenomenon. 
Keywords: Violence; Forms of violence; Conceptual analysis; Definitions of violence; Disciplinary and interdisciplinary approaches.

\section{Resumo}

Objetivos: apesar da abundante discussão acadêmica sobre violência, suas formas, atores, efeitos, entre outros elementos, surge uma pergunta quase permanentemente: do que estamos falando quando falamos de violência? Este artigo faz uma reflexão sobre a definição e análise do conceito de violência a partir de diferentes abordagens disciplinares e interdisciplinares, com o objetivo de tentar responder à pergunta: o que se entende por violência? Metodologia: foram consideradas algumas das contribuições de Antropologia, Ciência Política, Filosofia, Sociologia, Pesquisa para a Paz, Criminologia e Saúde Pública. Resultados: verificou-se que a maioria dos trabalhos que serviram de base para este estudo considera a violência como um elemento que delimita as interações sociais e não um ato irracional ou instintivo. E, dependendo da perspectiva da análise, estabelecem o grau de influência do cultural, simbólico, institucional e normativo em sua gestão e justificativa. Conclusões: conclui com um equilíbrio das vantagens e desvantagens da conveniência analítica da transição do termo violência para a expressão violência. Isso, considerando uma abordagem interdisciplinar que não apenas focaliza as manifestações físicas, mas também aborda a multidimensionalidade da violência e a estrutura criada pelas diferentes escalas de interação e afetação, transformando-a em um fenômeno social mutável e complexo.

Palavras-chave: Violência; Violências; Análise conceitual; Definições de violência; Abordagens disciplinares e interdisciplinares. 


\section{Introducción}

La discusión académica sobre la violencia se ha dividido en dos direcciones: la primera, concentra un gran número de trabajos que describen sus causas y cuantifican sus efectos. Algunas de estas investigaciones se han realizado sin una definición conceptual de su objeto de estudio. La segunda dirección se enfoca en analizar no sólo el origen sino las atribuciones de la violencia, con el objetivo de establecer una definición que permita comprender la realidad y delinear mecanismos para su atención y mitigación. En ambos casos se encuentra de manera constante una triada: poder, violencia y conflicto; conceptos que han sido objeto de múltiples análisis y abordajes desde distintas disciplinas. Ello, con el objetivo de responder ¿quién ejerce el poder o la violencia?, ¿Cómo las ejercen?, ¿Contra quién se ejercen? y ¿para qué se ejercen?

Sin embargo, existe una dificultad para su definición y, por ende, para su distinción. Es decir, la violencia se presenta como uno de los resultados del ejercicio del poder y del conflicto suscitado por ello. A su vez, el poder se profesa amparado por la violencia con el objetivo de mitigar las disputas.

¿Cómo definir a la violencia? La Real Academia Española define a la violencia como una cualidad (el que es violento) como una acción y efecto contra otra persona o contra uno mismo, así como una acción contra el natural modo de proceder $^{1}$. Esta definición deja más interrogantes que posibilidades reales para comprender el concepto, porque ¿cuál es este natural modo de proceder? ¿El qué está determinado por leyes o bien por normas sociales?, ¿Cuáles son los límites que establecen la naturalidad de las acciones? Es decir, la definición además de partir de una tautología establece una carga moral al pretender establecer un comportamiento natural de otro "antinatural”. Convirtiendo a la violencia en un elemento para la estigmatización y la discriminación de quien la ejerce -o es acusado de hacerlo-.

El presente trabajo es una Reflexión sobre la definición y análisis del concepto de la violencia desde distintos enfoques disciplinarios e interdisciplinarios, con el objetivo de intentar una respuesta para la pregunta ¿qué se entiende por violencia? En general, se abordarán las propuestas de autores pertenecientes a disciplinas que analizan a la violencia desde lo societal, es decir, que consideran tanto a las instituciones, las estructuras, sistemas y organizaciones sociales. También se presentarán tres propuestas interdisciplinarias, cuyos abordajes sobre la violencia permiten conceptualizar a la violencia como un complejo

1. Véase Diccionario de la Lengua Española, Violencia, https://dle.rae.es/?id=brdBvt6 (consultado 15/07/19). 
entramado de acciones, significados y comportamientos que se entretejen en la construcción de lo societal. Por último, en las conclusiones se presenta un balance de las propuestas abordadas con el objetivo de valorar la conveniencia analítica de transitar del término violencia a la expresión violencias, haciendo un balance de las ventajas -y desventajas- de dicho cambio.

\section{Metodología}

El análisis de la violencia se ha realizado desde distintos enfoques y disciplinas; por ejemplo, Elizabeth Stanko (2003) menciona que tales enfoques se pueden agrupar en tres principales vertientes: biologicistas, psicológicos y sociales. Los dos primeros tienen una aproximación subjetivista y, al segundo, se le puede considerar objetivista. Los enfoques biologicistas consideran a la violencia como un fenómeno natural e instintivo; por ello, las investigaciones se centran en la búsqueda de las causas genéticas, de la química cerebral y hormonal que determinan las conductas - por ejemplo, competencia por recursos naturales o económicos, la hostilidad, entre otros-. En el caso de las perspectivas psicológicas parten de un análisis del impacto de la ansiedad, frustración, agresión, privación, las experiencias traumáticas infantiles -abuso sexual, ausencia parental-y su interrelación con la curva de aprendizaje adquirida de los entornos familiares, comunitarios y escolares, entre otros abordajes. Por último, los enfoques sociales (considerados por la autora como estructuralistas) parten del análisis de la relación entre las estructuras sociales -relaciones de clase, género, simbólicas, entre otras- y las interacciones interpersonales (Stanko, 2003, p. 1).

De acuerdo con Siniša Malešević (2010), esta última perspectiva puede ser subdividida en tres grupos: globalista, elección racional y culturalista. La primera de ellas centra su análisis en el impacto que tienen las transformaciones macroestructurales de los últimos años del siglo XX, en las características de la violencia (Malešević, 2010, p. 59). En cuanto a la elección racional se enfoca en las dinámicas de las acciones y determinaciones individuales en la toma de decisión respecto a la violencia colectiva. Es decir, analiza la influencia que puede tener la racionalidad individual sobre la general al valorar los impactos y riesgos que la violencia tiene en términos económicos y políticos (Malešević, 2010, p. 60). Por último, la mirada culturalista se centra en el estudio de las diferencias religiosas, de las prácticas culturales, creencias, tradiciones, símbolos, rituales, entre otros procesos que pueden detonar y justificar acciones violentas en un contexto social determinado (Malešević, 2010, p. 64).

Teniendo como marco la clasificación propuesta por Stanko (2003) y Malešević (2010) para el análisis de la violencia en la presente sección, se abordarán de manera sucinta las miradas disciplinarias que comparten una 
perspectiva desde lo societal. Para ello, se realizó una selección de las principales propuestas de estudio de este fenómeno, enfocándose en aquellos autores que privilegian un análisis conceptual más que un estudio sobre casos concretos, además porque comparten una observación de la interacción entre las estructuras y las instituciones, más que de la conducta individual o comunitaria ${ }^{2}$.

Es necesario señalar, que el presente apartado no agota toda la producción académica de las disciplinas, sino que presenta una selección de los principales autores de cada una de ellas, quienes coinciden en enfocarse en tres aspectos: definir a la violencia, elementos que la componen o bien intervienen en su surgimiento, así como en las formas como se puede abordar su análisis. Ello, sin enfocarse en una secuencia cronológica, sino de análisis conceptual.

\section{Resultados}

\section{Violencia. Miradas disciplinarias}

\section{Antropología.}

La Antropología ha estudiado la violencia como parte de las interacciones sociales y el comportamiento humano. Uno de los objetivos de dichos estudios fue tratar de distinguir los límites entre las acciones violentas instintivas $-\mathrm{y}$ "biológicas"- de aquellas determinadas por las normas sociales. De esta manera, la violencia no sólo es un hecho sino también un proceso social con características y contextos históricos específicos. Por ello, el término adquiere un carácter polisémico dado que puede abarcar desde actos individuales, colectivos, organizados, espontáneos, rituales, legales o ilegales (Salmerón-Castro, 2017, p. 51).

De acuerdo con Elsa Blair-Trujillo (2009), los antropólogos han trabajado a la violencia mediante el análisis de su carácter fundador. Es decir, "todos los comienzos de las sociedades, de las civilizaciones y de los regímenes son periodos de violencia; los mitos del origen son todos ciclos de violencia. Pero una vez que la violencia ha tomado la forma en las instituciones (técnicas, normas, ritos), ella es convertida en fuerza creadora" (Blair-Trujillo, 2009, pp. 17-18), relacionándose de manera estrecha con el poder, el orden y el cambio social. Es decir, para algunos antropólogos, la violencia es considerada como una relación de poder situada en un contexto histórico y cultural específico, lo que hace que sus significados cambien en el tiempo y en el espacio. Así, cada cultura define

\footnotetext{
2. Es por ello que en el presente artículo no se ha considerado un abordaje desde la Psicología social; sin embargo, se han respetado las referencias de los autores seleccionados a dicha disciplina en aras de comprender su propuesta conceptual de la violencia.
} 
sus propios parámetros para explicar, ejercer y tolerarla, lo que convierte su uso en un ejercicio de negociación mediante el que se establece quiénes, cuándo y cómo deben ejercerla. Ejemplo de ello son los ritos (como medio simbólicos) y las normas (sociales o legales).

De acuerdo con René Girard (1977) la violencia es una fuerza generativa capaz de modificar las relaciones sociales a través de la reproducción de conflictos sociales. Por ello, su existencia ha sido controlada mediante la regulación de las interacciones humanas y de la mímesis que las media. Por ello, Girard señala que la violencia permanece invisible, sin embargo, se puede deducir en los mitos y rituales una vez que se han configurado como parte indispensable de las estructuras religiosas (Girard, 1977, p. 310). De esta manera, se convierte en sacra en tanto quede oculta y sólo conserve de manera visible su capacidad generativa $-\mathrm{y}$ reguladora- de las relaciones sociales. Ejemplo de ello son los sacrificios, cuya característica principal es la elección de una víctima, la cual al morir permite conservar el orden social. Es decir, su muerte desvía la atención -y la tensión- de la violencia colectiva hacia un ritual sangriento.

La violencia - de manera simultánea- es una exhibición de poder y en un elemento distintivo de un grupo social determinado ya que "los actos violentos tienen efectos profundos y duraderos sobre muchas personas que no tienen relación directa con ellos. La violencia extiende su eficacia en el tiempo y en el espacio y hace llegar su mensaje a muchas personas que no la sufren directamente" (Salmerón-Castro, 2017, p. 57). Sobre todo, al momento de desarrollar formas de canalizarla, es decir al crear rituales, competencias deportivas u otros mecanismos de orden social.

Ahora bien, la violencia también tiene la capacidad de "desnaturalizar" el orden social, sobre todo cuando los actos o comportamientos violentos trastocan las relaciones y las narrativas que los sustentan, lo cual provoca un proceso de rediseño de los mecanismos de interacción y, por ende, un cambio social ${ }^{3}$. Esta forma "desacralizada" de la violencia se relaciona con la apropiación y el uso de recursos que puede involucrar la eliminación del otro. Por lo anterior, desde la Antropología el análisis de la violencia se traslada de un estudio cuantitativo a una observación cualitativa que tenga en cuenta las características culturales específicas del grupo social en donde se presenta.

Debido a que la violencia presenta un carácter multifacético y en constante cambio, algunos antropólogos suelen emplear el término violencias para dar cuenta de las especificidades de las estructuras e interacciones sociales que las

3. Esta propuesta diferencia a la Antropología de otras disciplinas que emplean términos como "comportamiento o conducta antisocial", es decir, que hablan de elementos que rompen el orden y deben ser corregidos. Por el contrario, para la Antropología esta disolución presenta la oportunidad para modificar el orden social. 
originan y reproducen. Es decir, hacen énfasis en la descripción de un comportamiento social más que de una definición moral que suele emerger al usar el término en singular.

Este sucinto abordaje del análisis de la violencia desde la perspectiva antropológica no agota los distintos criterios y trabajos que se han elaborado en esta disciplina. Sin embargo, permite delinear un proceso de razonamiento enfocado en las interacciones sociales, así como en los símbolos y significados que las regulan. Elementos que se contrastarán con el siguiente apartado en donde se presenta el análisis de la violencia desde la Ciencia Política.

\section{Ciencia Política.}

En la Ciencia Política el análisis de la violencia se realiza desde la perspectiva de las interacciones políticas para la toma de decisión, el gobierno y el diseño de las instituciones que regulan a la sociedad, entre ellas el Estado. Por ejemplo, para Max Weber (1979) la violencia es el medio específico para el surgimiento del Estado, por ello éste reclama el monopolio y la concentración de la legitimidad en su uso. Es decir, “(...) a todas las demás asociaciones e individuos sólo se les concede el derecho a la violencia física en la medida en que el Estado lo permite. El Estado es la única fuente del “derecho” a la violencia” (Weber, 1979, pp. 83-84).

Así, el Estado regula las relaciones sociales mediante la autoatribución de la legitimidad en el uso de la violencia, así como la facultad para determinar y delimitar la acción violenta de los individuos o grupos que convivan en el territorio dominado por éste. De esta manera, va creando un entramado legal y legítimo para el control de la sociedad. Así, los individuos no sólo son despojados de la posibilidad de ejercer la violencia sino también son obligados a obedecer el marco legal que les impone dicho despojo, y ser sometidos al castigo, en caso de trasgredir dicho orden: "se acepta el recurso a la violencia a condición de que sea residual, absolutamente mínimo, subordinado al derecho y limitado materialmente por los derechos fundamentales" (Gallego-García, 2003. p. 91). De esta manera, la violencia legítima y se convierte en un elemento que origina y mantiene la estabilidad del Estado.

Coincidiendo con la reflexión de Weber, desde la filosofía política Walter Benjamin (1995) señala a la violencia como creadora del derecho. Es decir, a partir del triunfo de un grupo social en una contienda se establecen las nuevas reglas y normas de convivencia entre vencedores y vencidos. Por ello la violencia es una fuerza fundadora y al mismo tiempo un medio para conservar al derecho -y al Estado-(Benjamin, 1995, p. 41). A esta violencia Benjamin la denomina 
como mítica, la considera administrada y ejercida por el Estado mediante el derecho.

Benjamin rechaza la controversia acerca de la legitimidad en el monopolio estatal de la violencia y plantea que el derecho establece la distinción entre la violencia sancionada (la que le da origen) y la no sancionada (la que lo amenaza): "el interés del derecho por monopolizar la violencia respecto a la persona aislada no [tiene] como explicación la intención de salvaguardar fines jurídicos, sino más bien la de salvaguardar al derecho mismo" (Benjamin, 1995, p. 32). Por ello, el Estado a través del derecho implementa un entramado legal que le permiten "vedar" el uso de la violencia y conservar su monopolio sobre la misma.

Benjamin señala la existencia de una violencia "pura" y desligada a la imposición legal, a la cual denomina divina. Ésta destruye los límites impuestos por el derecho y redime a quien la ejerce mediante la exculpación:

[La] violencia divina no sólo es la tradición religiosa, (...) las manifestaciones de violencia divina no se definen por el hecho de que Dios mismo las ejercita directamente en los actos milagrosos, sino por el carácter no sanguinario, fulminante, purificador de la ejecución. En fin, por la ausencia de toda creación de derecho (Benjamin, 1995, p. 71).

Sin embargo, la violencia divina puede ser empleada como un mecanismo de legitimación, y, transmutarse en una violencia mítica como en el caso de algunos regímenes político-religiosos.

La filosofa Hannah Arendt (2006) también analiza la relación entre el poder y la violencia, señalando que ésta última es uno de los medios para mantener las estructuras de dominio frente a los retos individuales como los rebeldes o los delincuentes "quienes se niegan a ser superados por el consenso de la mayoría" (Arendt, 2006, p. 70). Es así, como la violencia adquiere un carácter instrumental que demanda de una justificación para su uso, sobre todo, considerando que se ejerce contra personas que son dañadas y expuestas de una manera deliberada para servir como un ejemplo o meta social. Por ello, se requiere de una racionalidad en su uso para generar la obediencia. Para Arendt (2006) la violencia y el poder son opuestos porque ésta surge cuando "el poder está en peligro, pero confiada a su propio impulso, acaba por hacer desaparecer al poder" (p, 77). Porque en presencia de la violencia represiva se genera el miedo social y una parálisis colectiva. Por ello, para Arendt la violencia es un instrumento de la reforma, porque permite externar los agravios y modificar el equilibro de la justicia.

Esta breve presentación del análisis de la violencia desde un enfoque político permite comprender la relación estrecha entre el surgimiento -y operación- del Estado con la violencia, pero ello no abarca todos los ámbitos de las relaciones 
sociales. Por ello, en el siguiente apartado se abordará la perspectiva filosófica de la violencia.

\section{Filosofía.}

La filósofa estadounidense Judith Butler (2006a, 2006b) señala que la violencia es la forma mediante la cual se muestra la vulnerabilidad del ser humano frente a los demás, ya que durante su ejercicio existe una entrega "sin control a la voluntad del otro, [por ello es] un modo por el que la vida misma puede ser eliminada por la acción deliberada del otro" (Butler, 2006b, p. 55). Por ello, es una forma de preservar el orden y el sentido del mundo.

Así, "la respuesta violenta es aquella que no requiere y no trata de conocer. Quiere reforzar lo que sabe [y] expurgar lo que lo amenaza (...) (Butler, 2006a, p. 60). Es una respuesta que refuerza los marcos culturales de lo "deseable", lo “aceptable” y lo "humano". Y todo aquello que lo cuestiona o lo subvierte debe ser eliminado para evitar la pérdida de los referentes sociales de comportamiento. De acuerdo con Butler, quien ejerce la violencia retira "la humanidad" a su víctima, lo que los transforma en seres irreales y por ende, las acciones que se realizan en su contra no provocan ningún daño, porque de antemano son vidas negadas (Butler, 2006b, p. 60). De esta manera, la violencia puede ser justificable y de manera simultánea convertirse en un elemento de la cotidianidad.

A partir de un análisis de las características de la sociedad en el siglo XXI, el filósofo surcoreano Byung Chul Han (2013) señala que la violencia física ha perdido legitimidad y, por ende, su ejercicio se oculta. En caso de que irrumpa en una sociedad, se le considera resultado de causas externas a ésta. Para comprender a la violencia propone clasificarla en dos tipos: macrofísica y microfísica. La primera de ellas "se manifiesta de modo expresivo, explícito, impulsivo e invasivo. La violencia microfísica, en cambio, se expresa de modo implícito e implosivo” (Han, 2013, p. 217). Han se enfoca en las características de este último tipo de violencia ya que a diferencia de la macrofísica que obliga a las víctimas a la pasividad, la microfísica se hace evidente mediante la hiperactividad de los sujetos.

Para Han, la violencia microfísica presenta tres características: se interioriza, se sirve del automatismo del hábito y se naturaliza. Esta triada actúa de manera interrelacionada, lo que dificulta a las personas cuestionar su ejercicio y, sobre todo, abstraerse de esta situación. Así, esta violencia es el resultado -y el origen- de la auto explotación, lo que hace de los sujetos víctima y victimario de manera simultánea. La sociedad presenta “opciones" de libertad que se convierten en prácticas de coacción, por ejemplo, cuando se glorifica el máximo rendimiento en diversos ámbitos como el consumo, lo laboral y lo comunicacional y la convierte en una "violencia positiva" en tanto que no se sustenta en la prohi- 
bición sino en el exceso de libertades. La consecuencia más aberrante es que no existe un punto final para este tipo de violencia (Han, 2013, p. 343). Además, esta "positividad" rechaza no sólo a la violencia física sino también al uso del lenguaje que niegue al otro, pero fomenta la atomización y el individualismo, lo cual tiene como resultado un proceso de descomposición de lo social.

A partir de esta sucinta Reflexión del análisis filosófico de la violencia es posible señalar que ésta no sólo es ejercida de manera física, sino que tiene múltiples dimensiones que tienen como consecuencia la negación de lo humano y de lo social. En el siguiente apartado, se abordará de manera abreviada el análisis sociológico de la violencia y sus consecuencias en las interacciones sociales.

\section{Sociología.}

El sociólogo alemán Niklas Luhmann (2006) realizó un análisis de la interacción entre el poder y la violencia. Su análisis parte de la distinción entre violencia legítima y violencia ilegítima, que son el producto de la evolución de la diferenciación social. Dice Luhmann (2006) que

En su forma legítima, la violencia (en la actualidad como violencia estatal) sirve para expulsar la violencia ilegítima. Con esta diferenciación, la violencia se caracterizará por la inclusión de lo excluido entonces (vista así) la legitimidad no es ningún concepto de valor sino precisamente esta inclusión de lo excluido -una paradoja pues, cuya solución se constituye como violencia de Estado- o su equivalente funcional" (p. 326).

Así, la violencia es un ejercicio de diferenciación funcional del Estado -es decir, del sistema político- del resto del sistema social. El cual se adjudica su ejercicio, así como la distinción respecto de las acciones violentas emprendidas por fuera del sistema político. Para ello, requerirá del ejercicio del poder, que es el que da origen y sentido al sistema político. Luhmann establece la distinción entre obligación y coerción como opciones en la operación del poder. El primero de ellos, se fundamenta en la neutralización de la voluntad del otro, es decir, el poder se convierte en la cesión del sometido hacia quien ostenta el poder, deseando que el poderoso evite la utilización de la coerción y de la violencia.

Por ello, la amenaza del uso de la violencia detona el proceso de coerción como segunda forma de operación del poder-, pero al ser una notificación, todavía permanece como una alternativa de uso desagradable. Ya que la advertencia de su uso se convierte en una alternativa para obtener aquello que la persuasión o la influencia no ha logrado. De acuerdo con Luhmann, al emplear la violencia física, el poder se anula; ello porque esta "se establece como el comienzo del siste- 
ma que conduce a la selección de reglas cuya función, racionalidad y legitimidad las hace independientes de las condiciones iniciales para la acción” (Luhmann, 1995, p. 94). De esta manera, al usarla será necesario establecer una nueva selección de reglas o condiciones para la operación del sistema. Y en esta reorganización, la violencia se convierte en la posibilidad de ordenamiento asimétrico y jerárquico, en donde el superior establece de manera evidente su predominio; sin embargo, también se crea un margen para que este orden pueda ser desafiado y, por ende, el portador del poder pierda su capacidad de ejercicio en la toma de decisión. Así, para Luhmann la interrelación entre violencia y poder determina la creación de este último, el cual, tendrá en la violencia un complemento pero no la base constante de su ejercicio porque a mayor uso de ésta se perderá la legitimidad del poder (Luhmann, 1995, p. 97).

Coincidiendo con esta línea de análisis, Pierre Bourdieu (1998) señala la existencia de una dominación simbólica que se produce mediante los esquemas de percepción y en las expectativas colectivas de comportamiento que modelan las relaciones, entre ellas, las de sumisión, que se sustentan en la violencia simbólica, la cual "se instituye a través de la adhesión que el dominado se siente obligado a conceder al dominador (por consiguiente, a la dominación)” (Bourdieu, 1998, p. 51). De esta manera, se moldean dichas relaciones transformándolas en relaciones afectivas, es decir, en un modo en el que los dominados aceptan como legítima su propia condición al considerarse en deuda por la "generosidad" del dominador.

La violencia simbólica se fundamenta en el habitus que genera -a su vezestructuras de dominación, un entramado complejo de interacción, que es difícil de modificar. Además, "La violencia declarada, física o económica y la violencia simbólica más refinada coexisten sin ninguna contradicción en todas las instituciones (...)” (Fernández, 2005. p. 10), estableciendo una relación paradójica: entre más alto sea el rechazo a la violencia física la simbólica se convierte en más aceptable. Esto, porque pese a ser "personalizada” -es decir radica en las características de la persona dominada- se convierte en un rasgo social y de ahí su capacidad para intricarse con el habitus y manifestarse en ámbitos tan "disímbolos" como la educación, la religión, la ciencia, las relaciones familiares, la política, entre otros. Los cuales coinciden en aportar formas simbólicas para interpretar el entorno, construir marcos de conocimiento, pero que ocultan el carácter impositivo de este entramado.

Además, la violencia simbólica se ejerce en los cuerpos de los dominados en las exigencias de comportamiento, omisión y aceptación en las interacciones sociales desde el vestido, el habla, las condiciones materiales de existencia (comer, dormir, reproducirse, etc.) hasta las formas de conducta aceptables en cada ámbito de interacción de dominación. Las acciones sobre los cuerpos son 
precedidas por la adopción de estructuras cognitivas, las cuales se refuerzan con la adopción física o somatizada de la violencia simbólica, que a su vez sostienen dichas estructuras. Así, la dominación establece un proceso continuo y sinérgico entre lo simbólico y lo físico, en donde las formas de la violencia adquieren un papel central para preservar el "orden natural de las cosas" en las interacciones sociales.

El filósofo y sociólogo esloveno Slavoj Žižek (2009) aborda en su análisis a la violencia. Señala que ésta se ha convertido en un lugar común debido al exceso de imágenes, discursos y símbolos mediante los cuales es presentada en los medios. Para su análisis, Žižek propone la distinción de tres tipos de violencia: subjetiva, simbólica y sistémica -estas dos últimas conforman la violencia objetiva-. Al primero de los casos, Žižek la considera la parte visible de la violencia "se le ve como una perturbación del estado de cosas "normal" y pacífico" (Žižek, 2009, p. 10). Es decir, se le considera como una explosión irracional y excesiva.

En contraste, las violencias simbólica y sistémica -que conforman a la violencia objetiva- constituyen ese estado "normal" de las cosas frente al cual la violencia subjetiva se presenta como una disrupción. La primera de ellas se encuentra en el lenguaje y en la conformación del universo de sentido mediante el cual se regula el comportamiento (Žižek, 2009, p. 10). En cuanto a la violencia sistémica es el resultado del funcionamiento de los sistemas político y económico. Žižek señala que la fascinación -y obsesión- por la violencia subjetiva distorsiona el análisis de la realidad porque se crea una distorsión basada en una "normalidad no violenta”, sin embargo, para Žižek la imposición de este parámetro de distinción es la más alta forma de violencia que se ejerce en la sociedad.

De manera similar, Michel Wieviorka (2003) define a la violencia como un mecanismo con funciones sociales y culturales cuya definición ha variado con el paso del tiempo. No sólo es un conjunto de prácticas objetivas (Wieviorka, 2003, p. 109) sino que es un elemento constitutivo de la subjetividad, es decir, de la capacidad de las personas de relacionarse con los demás. Así, la violencia se convierte en un portador de sentido, ya sea en ausencia o exceso. Por ejemplo, el martirio es una experiencia sobrecargada de sentido; en caso contrario, la acción violenta de los hooligans carece de éste.

Wieviorka propone clasificar a la violencia como infrapolítica y meta política. La primera de ellas se vincula a las actividades con fines económicos ilícitos como el tráfico de drogas, armas, órganos y seres humanos, es decir, es la privatización de la violencia (Wieviorka, 2009, p. 35) que se caracteriza por la impunidad en los actos cometidos, así como el control social sobre las poblaciones en donde las Mafias o las pandillas tienen su "territorio o zona de influencia". Además, la violencia infrapolítica se relaciona con el racismo y la xenofobia de- 
bido a que ambos comportamientos se encuentran en los márgenes de la política y de la aceptación social.

Wieviorka señala que la aparición de la violencia infra no implica el fin de la política. Por el contrario, su surgimiento puede ser el resultado de la diversificación de las actividades de los actores políticos. Así, de manera simultánea este tipo de violencia puede aparecer como ajena al Estado y, sin embargo, con un alto significado político (Wieviorka, 2009, p. 36). Por ejemplo, el caso de los narcotraficantes que realizan mejoras en la infraestructura de las comunidades de las que son originarios; lo cual les permite obtener un reconocimiento y apoyo social.

La violencia meta política implica su asociación a dimensiones e identidades culturales y religiosas mediante la radicalización de sectores de la población en contra de cierto orden. No reconoce fronteras ni concesiones sobre sus objetivos y significados. Quienes la ejercen pueden llegar a casos extremos como sacrificar su propia vida en aras de afirmar sus motivos (Wieviorka, 2009, p. 37). La violencia meta política también se vincula a la radicalización de individuos que se consideran a sí mismos como rechazados por la modernidad, o bien expulsados por la sociedad. Estas personas desarrollan un profundo sentimiento de injusticia y, por ende, la necesidad de resarcir o recuperar el orden social y político.

Ambos tipos de violencia se relacionan con la reducción del Estado, así como con procesos sociales y culturales. A diferencia de Weber, para Wieviorka, el Estado no determina la legitimidad de un acto violento, sino que esta radica en una densa red de valores sociales que cambian de manera gradual (Wieviorka, 2009, p. 45). Por ejemplo, las creencias religiosas otorgan significado a las acciones violentas, de manera simultánea que establecen expectativas de comportamiento tanto de las víctimas como de los victimarios.

\section{La multidimensionalidad de la violencia: miradas interdisciplinarias}

En la presente sección se abordarán tres propuestas analíticas sobre la violencia, las cuales, desde una mirada interdisciplinaria, pretenden comprender su complejidad tanto en las causas y efectos, como en los procesos de reproducción de las interacciones sociales enmarcadas en las acciones violentas. 


\section{Investigación para la paz 4 .}

En 1964, el trabajo de Johan Galtung (2008) marcó un cambio en el paradigma en la Investigación para la paz, al establecer una distinción entre distintos tipos de violencia y su correlación con la paz. Para Galtung la violencia es una conducta que se justifica como un acto de defensa o de prevención frente a las acciones de los demás; por ello, los sujetos no se consideran responsables al ejercerla. Además, es una venganza que posibilita obtener la satisfacción basada en el dolor del otro, en la perpetuación del "orgullo" de ser ganadores, o bien como un acto de "justicia" y expresión de la voluntad de fuerzas superiores (Galtung, 2008, pp. 278-279). Por ello, es una conducta y no sólo una parte de la naturaleza humana, ya que se requiere de circunstancias sociales que condicionen la realización de estas acciones.

Para analizar a la violencia -y sus contextos de surgimiento y operaciónGaltung propone clasificarla en tres grupos: directa, cultural y estructural. La primera de ellas es manifiesta, puede ser física y verbal. Los otros dos grupos permanecen latentes, es decir, no son percibidos como actos de violencia y, por ende, se justifican como parte de la normalidad. Así, el segundo tipo, la violencia estructural es intrínseca al sistema, se puede subdividir en política, económica, entre otras. La cultural es el soporte que legitima los tipos anteriores mediante la religión, la ley, la ideología, el lenguaje, el arte, la ciencia y la cosmología (Galtung, 1996, p. 36). De acuerdo con Galtung, este tipo de violencia se compone de las actitudes colectivas que por debajo de las actitudes individuales, determinan y distorsionan el comportamiento tanto de las personas como de su colectivo (Galtung, 2004, p.155).

Desde una perspectiva temporal, Galtung caracteriza a cada uno de los tres tipos de violencia como un acontecimiento (violencia directa), como un proceso en espiral, es decir con altas y bajas; (violencia estructural) y, por último, de largo alcance y lenta transformación (violencia cultural). La interacción de los tres tipos puede tener como consecuencia la anomia y la atomización social. Es decir, en un caso extremo una sociedad en conflicto puede enfrentarse a la disolución de las normas y valores sociales o bien comenzar un proceso de descomposición del tejido social y de la estructura del espacio público (Galtung, 2000, p. 185). Por ello, el análisis de la violencia tiene como núcleo la comprensión de la compleja sinergia entre sus distintos tipos, así como las relaciones que se establecen entre las personas y su entorno.

\footnotetext{
4. Emerge a mediados de la década de los cincuenta del siglo XX, con el objetivo de proponer metodologías de abordaje de los conflictos sociales e internacionales desde un enfoque interdisciplinar, abrevando de la Ciencia Política, las Relaciones Internacionales, la Antropología, la Psicología, entre otras disciplinas.
} 


\section{Criminologia 5 .}

Willem De Haan (2008) señala que la violencia es un término difícil de definir ya que es empleado para describir un enorme rango de comportamientos, emociones, situaciones y relaciones. Además, las distintas perspectivas de análisis -enfocadas ya sea en el victimario, las víctimas, acciones, efectos, causas, etc.dificultan establecer un concepto único. Por eso, para el autor la violencia es un fenómeno multifacético, socialmente construido y ambivalente (De Haan, 2008, p. 28), cuyas características se encuentran interrelacionadas haciendo compleja la elaboración de una definición única sobre la violencia.

En primer lugar, De Haan considera a la violencia como multifacética debido a que se presenta en diversas formas y en un amplio rango de contextos. Es decir, se le puede describir como física, verbal, individual, colectiva, interpersonal, institucional, nacional, internacional, simbólica y estructural. Puede ser ejercida en espacios públicos o privados; en cuanto a las víctimas pueden ser familiares, conocidos o extraños de los victimarios, quienes a su vez tienen como motivación el enojo, la impulsividad, hostilidad, entre otros, mientras que el motivo de la disputa puede ser instrumental o predatorio. Además, es necesario considerar el ámbito psicológico, social y material de la violencia (De Haan, 2008, p. 28). En segundo lugar, De Haan considera a la violencia como una construcción social debido a que la definición de quién y qué es violento varía de acuerdo con las especificidades socioculturales e históricas. Por ello, esta adquiere el carácter ambivalente en tanto que la heterogeneidad para definir el acto y el actor se reflejan al establecer las formas de sanción, legitimidad, institucionalización y transmisión cultural. De esta manera, es el contexto y la perspectiva social la que determinan si las acciones violentas son condenadas o admiradas.

Ante este panorama, De Haan establece dos tipos de perspectivas para el análisis de la violencia: restrictiva e inclusiva. La primera de ellas se enfoca solo en aquellas acciones que pueden ser percibidas por los sentidos (oído, vista, tacto, olfato y gusto). En cuanto a la segunda, considera que la violencia es un acto en contra de la humanidad y de la esencia del ser humano, por lo tanto, aplica tanto a sus cuerpos (violencia física) como a su habilidad para tomar decisiones (violencia psicológica), así como a las formas en como desde las instituciones (legales y morales) se restringe la vida (De Haan, 2008, p. 34). Desde la perspectiva inclusiva, el análisis de la violencia adquiere mayor dificultad y profundidad y, con ello, comprensión de un fenómeno de la realidad altamente complejo.

5. Desde un enfoque interdisciplinario analiza los procesos de criminalización enfocándose en los entornos sociales e institucionales que definen el crimen, las víctimas y las sanciones a dichas acciones. 


\section{Salud Pública ${ }^{6}$.}

A partir de una concepción de la complejidad de la violencia y con el objetivo de establecer líneas de análisis e intervención respecto a este fenómeno social, en el año 2003, la Organización Mundial de la Salud (OMS) presentó el Informe Mundial sobre la violencia y la salud. En el documento se define a la violencia como "el uso intencional de la fuerza o poder físico, de hecho o como amenaza, contra uno mismo, otra persona o comunidad, que cause o tenga muchas probabilidades de causar lesiones, muertes, daños psicológicos, trastornos del desarrollo o privaciones" (Organización Mundial de la Salud [OMS], 2003, p. 5). La definición de la OMS es amplia y se vincula a la discusión desarrollada en varias disciplinas acerca de la relación de la violencia y el poder. Es decir, no sólo se concentra en el acto mismo, sino en la intencionalidad al realizarlo, así como el contexto de las relaciones de poder en donde se encuentran insertas las víctimas y los victimarios. Lo que implica centrarse en el efecto global de la violencia en la salud y el bienestar de las personas, comunidades y sociedades (OMS, 2003, p. 6).

La propuesta de la OMS para el análisis de la violencia parte del estudio de la relación entre las causas y los efectos de la misma. Como primer punto señala que "aunque determinados factores biológicos y otros elementos individuales explican una parte de la predisposición a la agresión, más a menudo interactúan otros factores, familiares, comunitarios, culturales y otros agentes externos para crear una situación que favorece el surgimiento de la violencia” (OMS, 2003, p. 3). En cuanto a los efectos, se propone que el análisis vaya más allá de la cuantificación de las lesiones y muertes, ya que existen numerosas acciones que atentan contra la salud mental y las relaciones sociales. Por ello, se propone una tipología de la violencia basada en tres grupos: auto-infligida (como el suicidio), interpersonal y colectiva. Las cuales se encuentran interconectadas y dependiendo de los contextos sociales pueden ser justificadas y reproducidas; o en caso contrario, condenadas y prohibidas.

Las tres propuestas interdisciplinarias abordadas en este apartado, son una pequeña muestra de los esfuerzos por analizar la complejidad de la violencia desde el reconocimiento de su multidimensionalidad que no puede restringirse solo a sus efectos físicos. Por ello, se considera válido cuestionar si el propio término de violencia permite ponderar sus variaciones tanto en las acciones como en las víctimas.

\footnotetext{
6. Analiza los procesos que intervinieren en el bienestar físico y mental de individuos, comunidades, naciones y a nivel global, considerando una multiplicidad de factores que contribuyen -o bien truncan- dicha condición. Para ello, abreva de diversas disciplinas como las Ciencias Biológicas, Sociales, Económicas y de la conducta (entre ellas la Psicología social).
} 


\section{Conclusiones}

\section{¿Violencia o violencias? Apuntes conclusivos}

A partir de esta sucinta Reflexión sobre los trabajos que comparten un enfoque societal para analizar y definir la violencia, se delinearán algunos de los puntos en común entre éstos. En primer lugar, la mayoría coincide en señalarla como un producto de la construcción social, es decir, un elemento que delimita las interacciones sociales y no sólo como un acto instintivo -o irracional-. Al ejercerla se realiza un lento proceso de desmantelamiento de lo humano, ya que -en palabras de Butler (2006a) - se niega la posibilidad de reconocimiento del otro y su semejanza con quien actúa de manera violenta.

El sometimiento y la vulnerabilidad presentes en los actos de violencia se enmarcan en parámetros sociales e institucionales que establecen lo "normal" y lo "aceptable". Ello, porque de acuerdo con Girard (1977), la violencia se convierte en una fuerza creadora de sociedad que mediante ritos y patrones regula el comportamiento de los individuos. Punto en el que coinciden varios de los trabajos revisados, al señalar el vínculo estrecho entre violencia y poder. Porque las variables que aprueban -o rechazan- el comportamiento violento son definidas por este.

De acuerdo con Bourdieu (1998), quien ostenta el poder -persona o grupo social- además de establecer dichas variables de comportamiento, las transmite mediante una dominación simbólica que permite la reproducción social de estructuras de comportamiento y de los parámetros que valoran las conductas violentas desde una perspectiva moral y dicotómica. El grado extremo del proceso de apropiación de los sujetos con dichos parámetros establecidos por la dominación simbólica, pueden conducir a procesos de auto explotación. Según Han, estos emergen a partir de la "violencia positiva" que la sociedad del máximo rendimiento glorifica. Es decir, las personas establecen una dinámica frenética e -inacabable- de trabajo para consumir y comunicarse en un exceso de libertades.

La relación poder, violencia y dominación también es abordada por Luhmann, Weber, Benjamin y Arendt quienes ponen al Estado como la matriz de esta interacción, porque porta, define y delimita la violencia. Luhmann y Benjamin coinciden en señalar que la violencia es fundadora del sistema político - para el primero-o del derecho - para el segundo-. En el caso de Luhmann la relación poder y violencia se basa en un equilibro entre la amenaza y el empleo de ésta, que se pierde en el momento del uso de la violencia. En el caso de Benjamín, señala que la violencia es fundadora del derecho y, por ende, su sola existencia los convierte en equivalentes. Por último, Arendt señala que la violencia es un ins- 
trumento del poder y la dominación. Cabe aclarar que los tres autores se refieren únicamente a la acción física, quedando a la interpretación de los lectores, otro tipo de violencia relacionada con las estructuras e instituciones que esta genera.

Tanto Weber como Luhmann hablan de la legitimidad de la violencia, la cual parte de la distinción establecida por el grupo que ostenta el poder. Ya que este es quien determina la legalidad de los actos violentos, por ello, la "legitimidad" se convierte en un sustantivo cuyo significado varía de una sociedad a otra y, por lo tanto, definirla requiere de un ejercicio de revisión de los parámetros de comportamiento aceptados social y legalmente.

Wieviorka y Han cuestionan al Estado como el arquetipo de la violencia, en tanto portador de su monopolio legítimo. En especial, cuando su operación está en entredicho por la emergencia de nuevos actores legales e ilegales. En el primer caso, se encuentran el mercado y las empresas transnacionales que sobrepasan las capacidades normativas del Estado, disminuyen su posibilidad de acción y establecen límites alternos para el comportamiento aceptado socialmente (es decir, la violencia positiva de la que habla Han, 2013). Situación similar a los actos que realizan los actores ilegales vinculados a la violencia infrapolítica. Que se caracterizan por operar en los márgenes del Estado, pero con una gran aceptación social por lo que la legitimidad de la violencia radica en una densa red de valores sociales que cambian de manera gradual, los cuales, pueden cuestionar el entramado legal para la definición de las acciones violentas.

De esta manera, es posible señalar que no existe un poder único que monopolice la violencia, ya que el "poder es múltiple y polimorfo (...) es ubicuo, no porque el poder central se divida en una infinita variedad de ramas, sino porque múltiples relaciones de poder atraviesan, caracterizan y constituyen el cuerpo social, teniendo una estructura basilar por la cual circula"(Gallego-García, 2003, p. 86). Dando cabida a la multidimensionalidad de la violencia, a la cual se refieren Galtung, Žižek, De Haan y la OMS. Cada uno de ellos, menciona distintas características de los niveles de interacción -y afectación- de la violencia que van más allá de las acciones físicas. Así en las escalas individual, comunitaria, societal, cultural y estructural se presentan diversas formas de violencia que interactúan en un complejo entramado. Otro punto de coincidencia entre las propuestas revisadas, es el considerar a la violencia como un proceso, lo que implica que su análisis deba estimar tanto los contextos de origen como las consecuencias de los actos violentos. Lo que puede conducir a una aproximación a la compleja red de relaciones -y símbolos- sociales que tuvieron como consecuencia inicial un hecho de violencia física.

$\mathrm{Al}$ emplear el término violencia en la descripción de distintos fenómenos sociales provoca la pérdida de su sentido explicativo, restringiendo al concepto a una dimensión estrictamente cuantificable. Por ello, de acuerdo con De Haan, 
al transitar de un análisis restrictivo a uno inclusivo se adquiere mayor profundidad en la comprensión de un fenómeno social altamente complejo. Así, frente a la multidimensionalidad de sus manifestaciones, Stanko propone privilegiar la consideración de la violencia como un concepto fluido y mutable (Stanko, 2003, p. 3), en lugar de un concepto fijo que sólo hace referencia a las manifestaciones perceptibles.

Ello conduce a una disyuntiva, continuar con el uso de un término que ha perdido su capacidad explicativa debido a que al emplearlo casi de manera inmediata se hace referencia a las acciones físicas y, con ello, se deja de lado cualquier otro comportamiento; esto, puede "solventar" al incluir un apelativo que clarifique su significado, como violencia de género, social, escolar, familiar, psicológica, etc. O bien, de acuerdo con la propuesta del enfoque antropológico, emplear la expresión violencias y con ello, dar cuenta no sólo de la multidimensionalidad y complejidad de los distintos comportamientos que abarca el concepto, sino también de las diversas formas de definición y delimitación que cada sociedad establece. Por lo anterior, se propone el uso del término violencias para ampliar los parámetros de análisis de un fenómeno social mutable y complejo. Y con ello contribuir a clarificar este tipo de comportamientos insertos en el entramado de las interacciones sociales.

\section{Referencias}

Arendt, H. (2006). Sobre la violencia. Alianza Editorial.

Benjamin, W. (1995). Para una crítica de la violencia. Editorial Leviatán.

Blair-Trujillo, E. (2009). Aproximación teórica al concepto de violencia: avatares de una definición. Política y cultura, 32, 9-33. http://www.scielo.org.mx/ pdf/polcul/n32/n32a2.pdf [Consultado el 18 de octubre de 2014].

Bourdieu, P. (1998). La dominación masculina. Anagrama.

Butler, J. (2006a). Deshacer el género. Paidós. 
Butler, J. (2006b). Vida precaria: el poder del duelo y la violencia. Paidós.

De Haan, W. (2008). Violence as an Essentially Contested Concept. En S. Body-Gendrot y P. Spierenburg (Eds.). Violence in Europe. Historical and Contemporary Perspectives (pp. 27-40). Springer.

Fernández, J. M. (2005). La noción de violencia simbólica en la obra de Pierre Bourdieu: una aproximación crítica. Cuadernos de Trabajo Social, 18, 7-31. http://www.enlinea.cij.gob.mx/Cursos/Hospitalizacion/pdf/ PierreBourdieu.pdf

Gallego-García, G. M. (2003). Sobre el monopolio legítimo de la violencia. Nuevo Foro Penal, 66, 72-121. https://dialnet.unirioja.es/descarga/ articulo/3823123.pdf

Galtung, J. (1996). Peace by Peaceful Means. Peace and Conflict, Development and Civilization. International Peace Research Institute.

Galtung, J. (2000). 40 Conflicts; 40 Perspectives. En J. Galtung y C. G. Jacobsen (Eds.). Searching for Peace. The Road to TRANSCEND (pp. 122-190). Pluto Press.

Galtung, J. (2004). Transcend and Transform. An Introduction to Conflict Work. Pluto Press.

Galtung, J. (2008). The Transcend Approach to Simple Conflicts. En G. D. Paige y J. Evans Pim (Eds.). Global Nonkilling Leadership Fist Forum Proceedings (pp. 273-284). Center for Global Nonviolence, Matsunaga Institute for Peace, University of Hawaii.

Girard, R. (1977). Violence and the Sacred. The Johns Hopkins University Press.

Han, B. Ch. (2013). Topología de la violencia. Herder.

Luhmann, N. (1995). Poder. Anthropos.

Luhmann, N. (2006). La Sociedad de la Sociedad. Universidad Iberoamericana, Herder. 
Malešević, S. (2010). The Sociology of War and Violence. Cambridge University Press.

Organización Mundial de la Salud, OMS. (2003). Informe mundial sobre la violencia y la salud. Washington, D. C.: Organización Panamericana de la Salud, Oficina Regional para las Américas de la Organización Mundial de la Salud. https://www.who.int/violence_injury_prevention/violence/ world_report/en/summary_es.pdf

Salmerón-Castro, F. (2017). La Antropología y el análisis de la violencia. En L. Herrera-Lasso (Ed.). Fenomenología de la violencia: una perspectiva desde México (pp. 50-82). Siglo XXI Editores.

Stanko, E. (2003). Introduction. En E. Stanko (Ed.). The Meanings of Violence (pp. 1-14). Routledge.

Weber, M. (1979). El político y el científico. Alianza Editorial.

Wieviorka, M. (2003). The New Paradigm of Violence. En J. Friedman (Ed.). Globalization, the State and Violence (pp. 107-139). Altamira Press.

Wieviorka, M. (2009). Violence. SAGE Publications.

Žižek, S. (2009). Sobre la violencia. Seis reflexiones marginales. Paidós. 